Marquette University

e-Publications@Marquette

Biomedical Engineering Faculty Research and

Publications

Biomedical Engineering, Department of

4-1-2015

Sagittal Subtalar and Talocrural Joint Assessment With Weight-Bearing Fluoroscopy During Barefoot Ambulation

Ben McHenry

Marquette University, ben.mchenry@marquette.edu

Emily L. Exten

Medical College of Wisconsin

Jason Long

Cincinnati Children's Hospital Medical Center, Cincinnati

Brian Law

Medical College of Wisconsin

Richard Marks

Medical College of Wisconsin

See next page for additional authors

Accepted Version. Foot \& Joint International, Vol. 36, No. 4 (April 2015): 430-435. DOI. (C) 2015 Sage

Publications. Used with permission. 
Authors

Ben McHenry, Emily L. Exten, Jason Long, Brian Law, Richard Marks, and Gerald F. Harris 


\title{
Sagittal Subtalar and Talocrural Joint Assessment with Weight-Bearing Fluoroscopy During Barefoot Ambulation
}

\author{
Benjamin D. McHenry \\ Department of Biomedical Engineering, Marquette University \\ Milwaukee, WI \\ Emily L. Exten \\ Department of Orthopaedic Surgery \\ Division of Foot and Ankle Surgery \\ Medical College of Wisconsin \\ Milwaukee, WI \\ Jason Long \\ Cincinnati Children's Hospital Medical Center \\ Department of OT/PT and Orthopaedic Surgery \\ Cincinnati, $\mathrm{OH}$ \\ Brian Law \\ Department of Orthopaedic Surgery \\ Division of Foot and Ankle Surgery \\ Medical College of Wisconsin \\ Milwaukee, WI \\ Richard Marks \\ Department of Orthopaedic Surgery \\ Division of Foot and Ankle Surgery \\ Medical College of Wisconsin \\ Milwaukee, WI
}

Foot \& Ankle International, Vol 36, No. 4 (April 2015): pg. 430-435. DOI. This article is (C) SAGE Publications and permission has been granted for this version to appear in e-Publications@Marquette. SAGE Publications does not grant permission for this article to be further copied/distributed or hosted elsewhere without the express permission from SAGE Publications. 


\title{
Gerald Harris
}

\author{
Department of Biomedical Engineering, Marquette University \\ Milwaukee, WI
}

\begin{abstract}
Background: Identifying talar position during ambulation has proved difficult as the talus lacks palpable landmarks for skin marker placement and more invasive methodologies such as bone pins are not practical for most clinical subjects. A fluoroscopic motion system was used to track the talus and calcaneus, allowing kinematic analysis of the talocrural and subtalar joints.
\end{abstract}

Methods: Thirteen male subjects (mean age $22.9 \pm 3.0 \mathrm{yr}$ ) previously screened for normal gait were tested. A fluoroscopy unit was used to collect images at $120 \mathrm{fps}$ during stance. Sagittal motion of the talocrural and subtalar joints were analyzed.

Results: The inter-subject mean and standard deviation values for all 58 trials of 13 subjects are reported. Maximum talocrural joint plantarflexion of 11.2 degrees (4.3 degrees of standard deviation) occurred at $11 \%$ stance and maximum dorsiflexion of -6.9 degrees (5.6 degrees of standard deviation) occurred at $85 \%$. Maximum subtalar joint plantarflexion of 4.8 degrees ( 1.0 degrees of standard deviation) occurred at $96 \%$ stance and maximum dorsiflexion of -3.6 degrees (2.3 degrees of standard deviation) occurred at $30 \%$. Talocrural and subtalar range of motion values during stance were 18.1 and 8.4 degrees respectively. Conclusion: Existing fluoroscopic technology is capable of defining sagittal plane talocrural and subtalar motion during gait. These kinematic results compare favorably with more invasive techniques. This type of assessment could support more routine analysis of in vivo bony motion during gait.

Clinical Relevance: Fluoroscopic technology offers improved sagittal plane motion evaluation during weightbearing with potential application in patients with end stage ankle arthritis, postoperative ankle replacements and fusions, and orthotics and braces.

Keywords: gait analysis; hindfoot, subtalar joint, talocrural joint; ankle joint; biomechanics; weightbearing; fluoroscopy; sagittal motion

\section{Introduction}

Gait analysis is a proven method used to study lower extremity and foot and ankle kinematics. ${ }^{8}, 17,19,21,22,25$ The development of this technology has allowed us to evaluate several foot and ankle conditions and also compare treatments and postoperative outcomes. ${ }^{4,5,7,16,31}$

One limitation of current foot and ankle gait analysis models is their inability to uncouple talocrural and subtalar joint motion. These models use skin mounted markers attached to palpable bony landmarks to define the lower leg as one segment and the hindfoot as the adjacent segment. Hindfoot markers are usually only placed on the calcaneus. This is done to avoid interference with underlying tissue, to reduce skin motion artifact, and because the talus lacks palpable 
NOT THE PUBLISHED VERSION; this is the author's final, peer-reviewed manuscript. The published version may be accessed by following the link in the citation at the bottom of the page.

landmarks for accurate, consistent marker placement. ${ }^{25}$ Treating the talocrural and subtalar joint complex as one articulation does not allow for a true biomechanical analysis of either joint. This combining of motions only permits quantification of the summation of their individual contributions.

The ability to measure motion individually in these joints would allow us to further orthopedic knowledge in numerous areas. Such information would complement multiple recent studies reporting outcomes and ranges of motion. 3, 6, 9, 11,27-29, 35 A better understanding of individual and composite joint contributions to hindfoot motion during ambulation could improve treatment strategies. This includes kinematic effects of ankle arthritis and subsequent treatments, both operative and non-operative. Other applications include evaluation of joint motion resulting from pes planovalgus, tarsal coalition and instability. The quantitative effects following arthrodesis, arthroplasty, arthroscopy and open reduction internal fixation of the calcaneus, talus, pilon and malleolar fractures could be described. Further improvements could be anticipated when prescribing orthotics and shoe modifications for specific foot and ankle conditions.

An important consideration when obtaining joint motion data is the need to evaluate the activity of interest. Non-weight bearing studies of foot and ankle motion, for instance, do not provide an accurate assessment of weightbearing responses to load during gait. Weightbearing has been shown to alter measured joint ranges. Loading the ankle joint demonstrates increased sagittal motion. As shown by Lindsjo et al., there is a threefold increase in dorsiflexion when subjects were standing and weightbearing (32.5 \pm 6.9$)$ compared to supine with knees straight $(9.8 \pm 5.7)$ or flexed $(14.0 \pm 6.0)$. They also demonstrated a slight increase in plantarflexion of loaded ankles $(44.7 \pm 7.6)$ compared to supine with knees straight $(37.6 \pm 6.6)$ or flexed $(41.8 \pm 6.8) .{ }^{23}$

Bone pin based multi-segmental foot models are capable of individually measuring ankle and subtalar motion during weightbearing activities. Insertion of intra-cortical pins requires the assistance of an experienced orthopaedic surgeon and must be done under sterile operating conditions, and local anesthesia is used. After pin removal, subjects are given antibiotics and/or pain medication. While none of these studies report clinical complications, they all report subject pain and/or walking with a limp up to one week post analysis. 1, 2, 24, 33 To date, bone pin methodologies have been limited to research on healthy adult subjects.

Fluoroscopic imaging offers another method for tracking the ankle and subtalar joints. The dynamic radiographic method allows for the collection of images during motion. One of the first pioneering studies using fluoroscopy to track foot motion was done by Green et al. in $1975 .{ }^{14}$ Fluoroscopic images were captured on $16 \mathrm{~mm}$ film and anatomic bony motion (non-quantitative) was described as 
NOT THE PUBLISHED VERSION; this is the author's final, peer-reviewed manuscript. The published version may be accessed by following the link in the citation at the bottom of the page.

subjects moved their foot from maximal pronation to maximal supination. Since the work of green, a number of studies applying fluoroscopic techniques to the foot and ankle appear in the literature. In 2005, Wearing et al. used single plane fluoroscopy and static positioning to determine errors in measuring sagittal arch kinematics of the human foot using dried bone pieces. ${ }^{32}$ In 2007, Wrbaskic and Dowling used single plane fluoroscopic imaging $(60 \mathrm{~Hz}$.) to report on the deformable characteristics of the human foot, but did not include kinematic results. ${ }^{34}$ In a 2011 study, Shultz et al. quantified skin motion artifact error of the foot using single plane fluoroscopy and quasi-static foot positioning. ${ }^{30}$ In one of the most recent studies, Iaquinto et al. tracked the dynamic motion of beads embedded in plastic at known locations to validate a biplane fluoroscopy system for quantifying foot kinematics. ${ }^{18}$ Two articles in the literature use fluoroscopy to report ankle joint kinematics, but both use static positioning of the foot. In a 2000 study by Komistek et al., sagittal plane ankle kinematics were reported for ten subjects between static dorsiflexion and static plantarflexion positions. ${ }^{20}$ Because of the static nature of the study methodology, only ranges of motion could be reported. In a bi-planar (dualorthogonal fluoroscopy) study by de Asla et al., talocrural, subtalar, and tibiocaneal (calcaneus with respect to tibia) kinematics were reported among three static positions (heel strike, mid-stance, and toe-off). ${ }^{10}$ Similar to the Komistek et al. study, static positioning of the foot limited the results to ranges of motion.

The goal of this study was to apply a fluoroscopic motion analysis system to assess talocrural and subtalar joint motion in the sagittal plane during the stance phase of gait.

\section{Materials and Methods}

In this Institutional Review Board (IRB) approved study, 13 male subjects (mean age $22.9 \pm 3.0 \mathrm{yr}$, mean weight $77.2 \pm 6.9 \mathrm{~kg}$, mean height $178.2 \pm 3.7$ $\mathrm{cm})$, previously screened for exclusion criteria were tested over a four month period. Exclusion criteria included any significant injury to the foot and/or ankle or any previous lower extremity surgery (bilateral).

A standard fluoroscopic system was synchronized with an existing Vicon, Nexus motion analysis system (Vicon Motion Systems, Inc., Oxford, UK). The fluoroscopy unit (OEC 9000, GE, Fairfield, CT) was modified so that the emitter and image intensifier (II) were detached and mounted on opposite sides of the width of the walkway (Figure 1).

The right leg and foot of each subject were instrumented with six reflective markers ( $d=16 \mathrm{~mm}$ ) in accordance with the distal portion of the Plug-In-Gait model and an additional marker (Table 1). Simultaneous motion analysis and fluoroscopic data were collected (120 fps) as subjects walked at a self-selected 
NOT THE PUBLISHED VERSION; this is the author's final, peer-reviewed manuscript. The published version may be accessed by following the link in the citation at the bottom of the page.

pace along the eight meter custom walkway. The fluoroscopic system was manually activated at heel strike/initial contact and de-activated just after toe-off. During fluoroscopic data collection radiation levels were set from 90-110 kVp, and 0.5-1.7 $\mathrm{mA}$ depending on patient-specific image quality analyses. These radiation levels were confirmed by the radiation safety department prior to application. Each subject completed five barefoot trials with fluoroscopic imaging as approved by the IRB. Due to foot misplacements within the capture volume during radiation exposure, seven of the 13 subjects had four acceptable trials. Following dynamic data collection, subjects were escorted to a nearby $x$-ray suite where a single limb support barefoot $x$-ray was taken of their right foot placed at the same foot progression angle observed during foot flat of the dynamic image collection.

The fluoroscopic system was used to analyze the talocrural and subtalar joint motion. This required tracking of tibial, talar and calcaneal position during stance. The talus and calcaneus were tracked directly in each of the collected fluoroscopic images. Two points of interest per bone (Figure 2) were translated from pixel coordinates to motion analysis global coordinates using the known external marker (Table 1) location data. These translated points of interest were defined in the sagittal plane of the foot and were then used to describe local coordinate systems for the talus and calcaneus. External markers (medial/lateral malleoli and medial/lateral femoral epicondyles) were used to define the tibial local coordinate system as only the very distal end of the tibia was fluoroscopically visible for much of stance. Although only talocrural and subtalar motion is reported in the current study, the external marked based model is able to track motion proximal to the ankle. ${ }^{12,13}$ Kinematic analysis was completed by using the International Society of Biomechanics (ISB) recommended Joint Coordinate Method, with motion being reported as distal segment movement with respect to proximal. ${ }^{15}$ This kinematic model was additionally applied to the static lateral $x$-ray which defined neutral position. Kinematic results are reported relative to this neutral position.

\section{Results}

The talocrural joint increases to maximum plantarflexion during loading response followed by dorsiflexion motion during mid and terminal stance (Figure 3). Maximum talocrural dorsiflexion occurs at the beginning of preswing followed by plantarflexion towards swing phase. The subtalar joint is in a neutral position at heel strike followed by dorsiflexion into mid-stance. Maximum subtalar dorsiflexion occurs during mid-stance, though little motion occurs again until the middle of terminal stance at which point plantarflexion occurs for the rest of stance. The maximum talocrural plantar and dorsiflexion kinematics occur at $11 \%$ and $85 \%$ stance respectively (Table 2 ). The maximum subtalar plantar and dorsiflexion kinematics occur greater than $96 \%$ and $30 \%$ stance respectively. 
NOT THE PUBLISHED VERSION; this is the author's final, peer-reviewed manuscript. The published version may be accessed by following the link in the citation at the bottom of the page.

\section{Discussion}

Existing technology that allows evaluation of sagittal talocrural and subtalar motion supports a better understanding of in vivo joint kinematics. Brodsky et al used the Milwaukee Foot Model ${ }^{19}$ to evaluate the hindfoot motion of 46 patients following total ankle arthroplasty with a STAR prosthesis. This study found decreased hindfoot motion in patients following ankle arthroplasty in both the sagittal and coronal planes. The authors highlighted the need to better understand hindfoot and more specifically subtalar joint pathology present both before and after surgery. They questioned whether subtalar arthritis existed prior to surgery as a result of the end stage ankle arthritis and subsequent gait abnormalities, or if it developed following the ankle arthroplasty. ${ }^{6}$

The only current foot model that reports talocrural and subtalar motion during gait uses direct measurement methods with implanted bone pins. Kinematic results from this methodology compare favorably with the current fluoroscopic model, both in morphology and range of motion. Fluoroscopic maximum talocrural plantar and dorsiflexion kinematics occur at $11 \%$ and $85 \%$ stance respectively, with a range of motion of 18.1 degrees (Figure 3, Table 2). Sagittal plane talocrural joint kinematic graphs from two recent bone pin studies agree with these results, showing maximum plantarflexion occurring before $15 \%$ of stance, and maximum dorsiflexion occurring after $80 \%$ with range of motion values between 11.3 and 18.7 degrees. ${ }^{1}{ }^{24}$ Fluoroscopic maximum talocrural plantar and dorsiflexion kinematics occur at the end of preswing and 30\% stance respectively, with a range of motion of 8.4 degrees (Figure 3, Table 2). The aforementioned bone pin studies show varied ranges of maximum plantar and dorsiflexion, but correspond to a range of motion between 2.8 and 8.8 degrees. ${ }^{1,24}$

Current external marker based multi-segmental foot models report only hindfoot/calcaneal segment motion with respect to a lower leg segment. ${ }^{8}, 17,19,21,22$ Results from these models cannot be directly compared to the current fluoroscopic results because of their inability to differentiate between talocrural and subtalar motion. The 2008 bone pin study, however, does report calcaneal motion with respect to tibia and can be directly compared to the external marker based models. In Lundgren's bone pin study the average tibial-calcaneal sagittal plane ROM is 17 degrees for six subjects. ${ }^{24}$ The aforementioned external marker based models report an average of 18 degrees of sagittal plane hindfoot/calcaneal segment motion on a total of 39 feet. $^{8}, 17,19,21,22$ While the fluoroscopic model does not directly measure calcaneal motion with respect to tibia, the talocrural and subtalar motion does compare favorably to the 2008 bone pin study.

Current study limitations include a narrow sample of adult male subjects aged 18 to 28 with no reported gait deficiencies or prior bony foot injury. While the 
NOT THE PUBLISHED VERSION; this is the author's final, peer-reviewed manuscript. The published version may be accessed by following the link in the citation at the bottom of the page.

results of this pilot study are promising, further studies should be conducted using a larger cohort of healthy as well as pathologic gait populations. The current study is also limited to a single plane (sagittal) analysis of hindfoot motion components. A further limitation is the use of ionizing radiation with current levels estimated at ten $\mu \mathrm{Sv} /$ trial. United States Nuclear Regulatory Commission (USNRC) whole body annual occupational limits are five rems $(50,000 \mu \mathrm{Sv})$.

In conclusion, sagittal fluoroscopic imaging compares favorably to results from bone pin implants without the need for invasive procedures. With regard to motion contribution, both talocrural and subtalar joints exhibit sagittal motion throughout stance. Continued assessment of talocrural and subtalar ranges of motion during stance may further our understanding of hindfoot biomechanics during gait with implications for improved clinical applications. These clinical applications include evaluation of joint motion in pathologic conditions such as arthritis, pes planovalgus, tarsal coalition, and instability. The postoperative motion of the ankle and subtalar joints may be investigated following arthrodesis, arthroplasty, arthroscopy, and open reduction internal fixation of calcaneus, talus, pilon, and ankle fractures. Nonoperative treatment of foot and ankle conditions with custom fabricated orthoses and shoe modifications may also benefit from the development of this system. Further applications would include higher speed analyses of running kinematics and footwear choices among recreational to elite athletes.

\section{References}

1. Arndt, A., P. Westblad, I. Winson, et al., Ankle and subtalar kinematics measured with intracortical pins during the stance phase of walking. Foot Ankle Int, 2004. 25(5): p. 357-64.

2. Arndt, A., P. Wolf, A. Liu, et al., Intrinsic foot kinematics measured in vivo during the stance phase of slow running. J Biomech, 2007. 40(12): p. 2672-8. doi: 10.1016/j.jbiomech.2006.12.009

3. Barton, T., F. Lintz and I. Winson, Biomechanical changes associated with the osteoarthritic, arthrodesed, and prosthetic ankle joint. Foot Ankle Surg, 2011. 17(2): p. 52-7. doi:10.1016/j.fas.2011.01.010

4. Brodsky, J.W., Preliminary gait analysis results after posterior tibial tendon reconstruction: a prospective study. Foot Ankle Int, 2004. 25(2): p. 96-100

5. Brodsky, J.W., B.S. Baum, F.E. Pollo, et al., Prospective gait analysis in patients with first metatarsophalangeal joint arthrodesis for hallux rigidus. Foot Ankle Int, 2007. 28(2): p. 162-5. doi:10.3113/FAI.2007.0162

6. Brodsky, J.W., S.C. Coleman, S. Smith, et al., Hindfoot motion following STAR total ankle arthroplasty: a multisegment foot model gait study. Foot Ankle Int, 2013. 34(11): p. 1479-85. doi:10.1177/1071100713494381

7. Canseco, K., J. Long, R. Marks, et al., Quantitative characterization of gait kinematics in patients with hallux rigidus using the Milwaukee foot model. J Orthop Res, 2008.

26(4): p. 419-27. doi:10.1002/jor.20506

Foot \& Ankle International, Vol 36, No. 4 (April 2015): pg. 430-435. DOI. This article is C SAGE Publications and permission has been granted for this version to appear in e-Publications@Marquette. SAGE Publications does not grant permission for this article to be further copied/distributed or hosted elsewhere without the express permission from SAGE Publications. 
NOT THE PUBLISHED VERSION; this is the author's final, peer-reviewed manuscript. The published version may be accessed by following the link in the citation at the bottom of the page.

8. Carson, M.C., M.E. Harrington, N. Thompson, et al., Kinematic analysis of a multisegment foot model for research and clinical applications: a repeatability analysis. J Biomech, 2001. 34(10): p. 1299-307. doi:S0021929001001014 [pii]

9. Coetzee, J.C. and M.D. Castro, Accurate Measurement of Ankle Range of Motion after Total Ankle Arthroplasty. Clinical Orthopaedics and Related Research, 2004. 424: p. 27-31. doi:10.1097/01.blo.0000132180.69464.84

10. de Asla, R.J., L. Wan, H.E. Rubash, et al., Six DOF in vivo kinematics of the ankle joint complex: Application of a combined dual-orthogonal fluoroscopic and magnetic resonance imaging technique. Journal of orthopaedic research, 2006. 24(5): p. 1019-1027

11. Flavin, R., S.C. Coleman, S. Tenenbaum, et al., Comparison of gait after total ankle arthroplasty and ankle arthrodesis. Foot Ankle Int, 2013. 34(10): p. 1340-8. doi: $10.1177 / 1071100713490675$

12. Graf, A., S. Hassani, J. Krzak, et al., Long-term outcome evaluation in young adults following clubfoot surgical release. J Pediatr Orthop, 2010. 30(4): p. 379-85. doi:10.1097/BPO.0b013e3181d8fbd7

13. Graf, A., K.W. Wu, P.A. Smith, et al., Comprehensive review of the functional outcome evaluation of clubfoot treatment: a preferred methodology. J Pediatr Orthop B, 2012. 21(1): p. 20-7. doi:10.1097/BPB.0b013e32834dd239

14. Green, D., T. Sgarlato and M. Wittenberg, Clinical biomechanical evaluation of the foot: a preliminary radiocinematographic study. Journal of the American Podiatry Association, 1975. 65(8): p. 732

15. Grood, E.S. and W.J. Suntay, A joint coordinate system for the clinical description of three-dimensional motions: application to the knee. J Biomech Eng, 1983. 105(2): p. $136-44$

16. Hahn, M.E., E.S. Wright, A.D. Segal, et al., Comparative gait analysis of ankle arthrodesis and arthroplasty: initial findings of a prospective study. Foot Ankle Int, 2012. 33(4): p. 282-9. doi:10.3113/FAI.2012.0282

17. Hunt, A.E., R.M. Smith, M. Torode, et al., Inter-segment foot motion and ground reaction forces over the stance phase of walking. Clin Biomech (Bristol, Avon), 2001. 16(7): p. 592-600. doi:S0268003301000407 [pii]

18. 18. Iaquinto, J.M., R. Tsai, D.R. Haynor, et al., Marker-based validation of a biplane fluoroscopy system for quantifying foot kinematics. Medical engineering \& physics, 2014. 36(3): p. 391-396

19. Kidder, S.M., F.S. Abuzzahab, Jr., G.F. Harris, et al., A system for the analysis of foot and ankle kinematics during gait. IEEE Trans Rehabil Eng, 1996. 4(1): p. 25-32

20. Komistek, R.D., J.B. Stiehl, F.F. Buechel, et al., A determination of ankle kinematics using fluoroscopy. Foot \& Ankle International, 2000. 21(4): p. 343-350

21. Leardini, A., M.G. Benedetti, L. Berti, et al., Rear-foot, mid-foot and fore-foot motion during the stance phase of gait. Gait Posture, 2007. 25(3): p. 453-62. doi:S09666362(06)00160-3 [pii] 10.1016/j.gaitpost.2006.05.017

22. Leardini, A., M.G. Benedetti, F. Catani, et al., An anatomically based protocol for the description of foot segment kinematics during gait. Clin Biomech (Bristol, Avon), 1999. 14(8): p. 528-36. doi:S0268-0033(99)00008-X [pii]

23. Lindsjo, U., G. Danckwardt-Lilliestrom and B. Sahlstedt, Measurement of the motion range in the loaded ankle. Clin Orthop Relat Res, 1985(199): p. 68-71 
24. Lundgren, P., C. Nester, A. Liu, et al., Invasive in vivo measurement of rear-, midand forefoot motion during walking. Gait \&amp; Posture, 2008. 28(1): p. 93-100

25. MacWilliams, B.A., M. Cowley and D.E. Nicholson, Foot kinematics and kinetics during adolescent gait. Gait Posture, 2003. 17(3): p. 214-24. doi:S0966636202001030 [pii]

26. Perry, J., Gait analysis: normal and pathological function. Slack, Thorofare, NJ, 1992

27. Queen, R.M., J.C. De Biassio, R.J. Butler, et al., J. Leonard Goldner Award 2011: changes in pain, function, and gait mechanics two years following total ankle arthroplasty performed with two modern fixed-bearing prostheses. Foot Ankle Int, 2012. 33(7): p. 535-42. doi:10.3113/FAI.2012.0535

28. Rouhani, H., J. Favre, K. Aminian, et al., Multi-segment foot kinematics after total ankle replacement and ankle arthrodesis during relatively long-distance gait. Gait Posture, 2012. 36(3): p. 561-6. doi:10.1016/j.gaitpost.2012.05.010

29. Saltzman, C.L., R.A. Mann, J.E. Ahrens, et al., Prospective controlled trial of STAR total ankle replacement versus ankle fusion: initial results. Foot Ankle Int, 2009. 30(7): p. 579-96. doi:10.3113/FAI.2009.0579

30. Shultz, R., A. Kedgley and T. Jenkyn, Quantifying skin motion artifact error of the hindfoot and forefoot marker clusters with the optical tracking of a multi-segment foot model using single-plane fluoroscopy. Gait \& posture, 2011. 34(1): p. 44-48

31. Smith, S.M., S.C. Coleman, S.A. Bacon, et al., Improved ankle push-off power following cheilectomy for hallux rigidus: a prospective gait analysis study. Foot Ankle Int, 2012. 33(6): p. 457-61. doi:10.3113/FAI.2012.0457

32. Wearing, S.C., J.E. Smeathers, B. Yates, et al., Errors in measuring sagittal arch kinematics of the human foot with digital fluoroscopy. Gait \& posture, 2005. 21(3): p. 326-332

33. Westblad, P., T. Hashimoto, I. Winson, et al., Differences in ankle-joint complex motion during the stance phase of walking as measured by superficial and boneanchored markers. Foot Ankle Int, 2002. 23(9): p. 856-63

34. Wrbaškić, N. and J. Dowling, An investigation into the deformable characteristics of the human foot using fluoroscopic imaging. Clinical Biomechanics, 2007. 22(2): p. 230-238

35. Zaidi, R., S. Cro, K. Gurusamy, et al., The outcome of total ankle replacement: a systematic review and meta-analysis. Bone Joint J, 2013. 95-B(11): p. 1500-7. doi:10.1302/0301-620X.95B11.31633 
NOT THE PUBLISHED VERSION; this is the author's final, peer-reviewed manuscript. The published version may be accessed by following the link in the citation at the bottom of the page.

\section{TABLES}

Table 1: External marker locations.

\begin{tabular}{ll}
\hline Marker Location & Marker Name \\
\hline Calcaneal tuberosity & M1 \\
Head of the $2^{\text {nd }}$ metatarsal & M2 \\
Medial malleolus & M3 \\
Lateral malleolus & M4 \\
Medial femoral epicondyle & M5 \\
Lateral femoral epicondyle & M6 \\
\hline
\end{tabular}

Table 2: Talocrural and subtalar kinematics during stance phase.

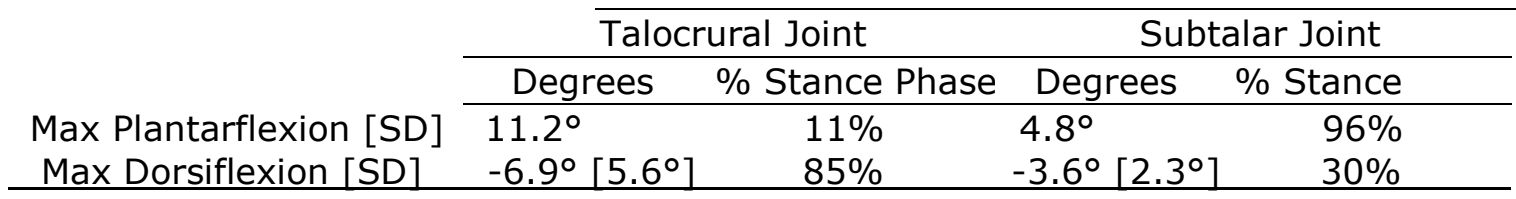

\section{Figures}

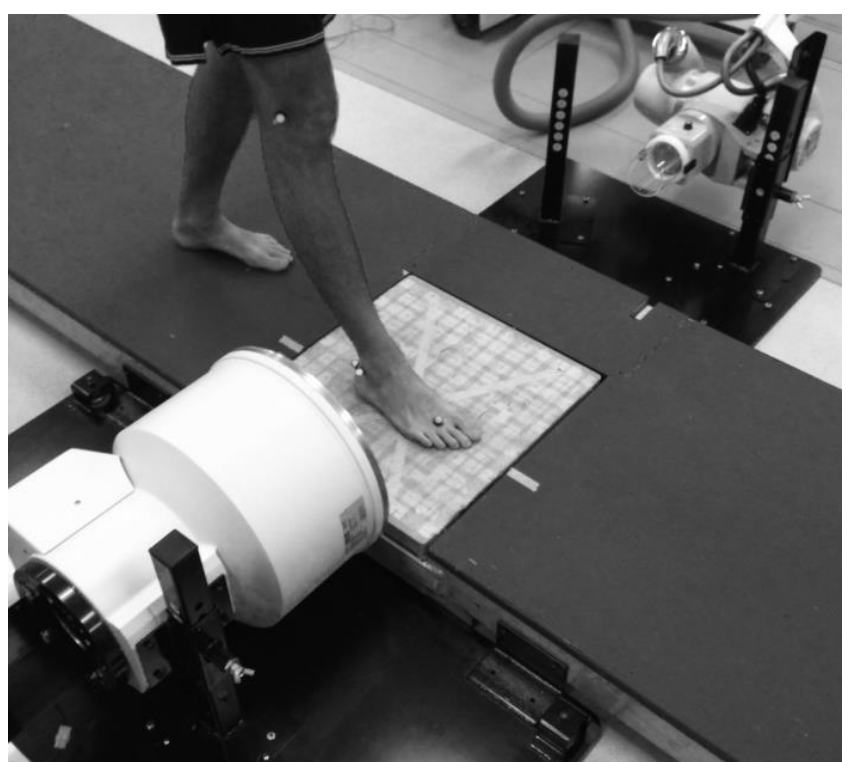

Figure 1. System configuration showing the walkway, emitter (farside), and image intensifier (nearside). Also shown is typical foot placement during image collection. 
NOT THE PUBLISHED VERSION; this is the author's final, peer-reviewed manuscript. The published version may be accessed by following the link in the citation at the bottom of the page.

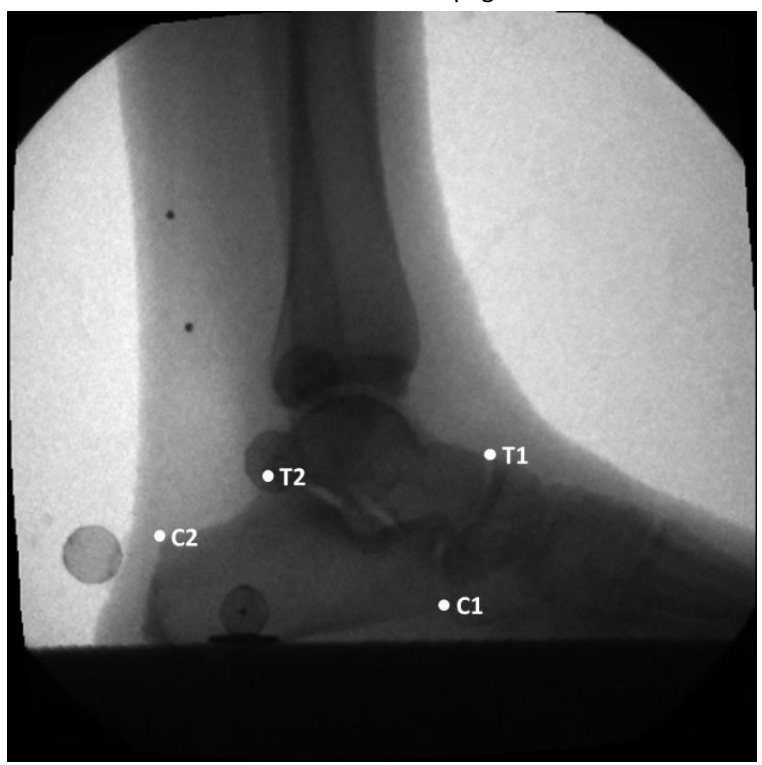

Figure 2. Typical fluoroscopic image showing the points of interested used to fluoroscopically track the talus and calcaneus (Talus: T1 - superior border of the talonavicular joint, T2 - posterior talar process; Calcaneus: C1 - inferior aspect of the calcaneocuboid joint, $\mathrm{C} 2$ - superior aspect of the posterior tuberosity of the calcaneus).
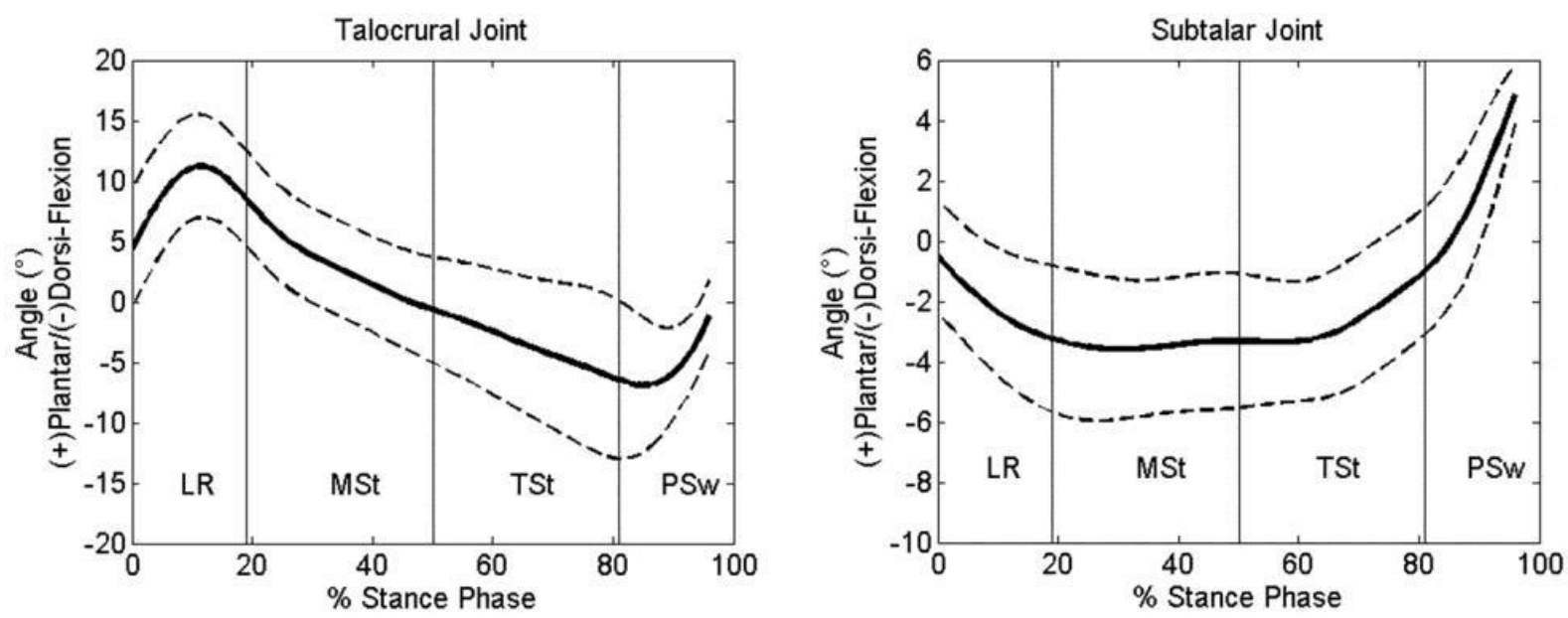

Figure 3. Talocrural (left) and Subtalar (right) plantar/dorsiflexion angles. Solid lines represent mean of all 13 subject trials. Dashed lines represent mean \pm 1 SD. Results are sub-divided into LR: loading response (initial contact through contralateral toe-off), MSt: mid-stance (contralateral toe-off through ipsilateral heel-rise), TSt: terminal stance (ipsilateral heel-rise through contralateral initial contact), and PSw: preswing (contralateral initial contact through ipsilateral toe-off). ${ }^{26}$ Missing data between $97-100 \%$ of stance corresponds to the foot vacating the field of view. 